\title{
Preparation and Physicochemical Characterization of Solid Dispersion of Irbesartan with Poloxamer 188
}

\author{
Fifi Harmely ${ }^{1}$, Salman Umar ${ }^{2}$, Yufri Aldi ${ }^{2}$, Ellyza Nasrul ${ }^{1}$, Erizal Zaini ${ }^{2 *}$ \\ ${ }^{1}$ Department of Biomedical Sciences, Faculty of Medicine, Andalas University, Padang 25163, Indonesia; ${ }^{2}$ Faculty of Pharmacy, \\ Andalas University, Padang 25163, Indonesia
}

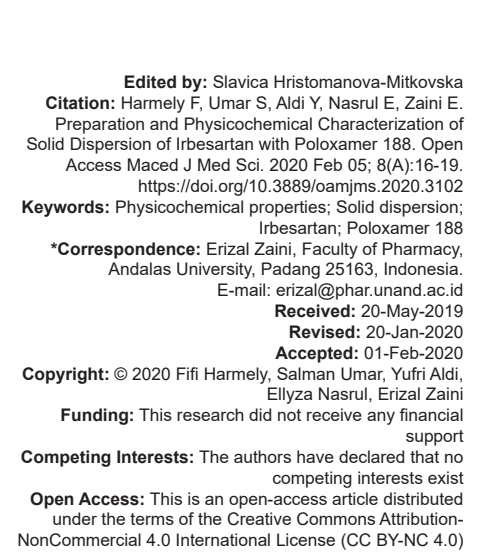

\begin{abstract}
AIM: The aim of the present study was to prepare and characterize the solid dispersion of poorly soluble drugs irbesartan with hydrophilic polymer poloxamer 188 by solvent co-evaporation technique.

METHODS: The ratio of irbesartan to poloxamer 188 in solid dispersion system was $1: 0.5 ; 1: 1$; and $1: 3$ Physicochemical properties characterization was analyzed by X-ray powder diffraction, Fourier-transform infrared (FT-IR) spectroscopy, differential thermal analysis, and scanning electron microscopy (SEM). The in vitro dissolution rate profile of solid dispersion was performed by Type II United States Pharmacopeia dissolution testing apparatus.

RESULTS: The results of the X-ray powder diffraction pattern showed that the degree of crystallinity of irbesartan crystalline phase reduced in a solid dispersion system. FT-IR spectra indicate that there is no chemical interaction between irbesartan and poloxamer 188 in a solid dispersion system. SEM microscopy demonstrated a homogenous phase of a solid dispersion system with distinct crystal habit. In general, in vitro dissolution rate of all solid dispersions was higher than intact irbesartan. The highest percentage of dissolved irbesartan was the solid dispersion of irbesartan-poloxamer $188(1: 0.5 \mathrm{w} / \mathrm{w})$.
\end{abstract}

CONCLUSIONS: The study concludes that the solid dispersion can be applied to improve the dissolution rate of irbesartan.

\section{Introduction}

Irbesartan (IBS) is 2-butyl-3-[[2-(1H-tetrazole5-yl)(1,1-biphenyl)-4-yl]methyl]-1,3 diazaspiro[4,4]non1-en-4-one. IBS, a specific competitive blocker of the angiotensin II receptor, is orally used for the treatment of hypertension as well as renal disease in hypertensive diabetic patients [1]. IBS has a low solubility in water $(0.00884 \mathrm{mg} / \mathrm{mL})$ with $26 \%$ oral bioavailability. It belongs to Biopharmaceutics Classification System (BCS) Class II drugs. The bioavailability of active pharmaceutical ingredients (API) is significantly related to the BCS. More than $40 \%$ of API in the market are categorized as BCS Class II (low solubility and high permeability) [2]. The poorly soluble drugs often show low gastrointestinal absorption due to low drug solubility in the gastrointestinal fluid and dissolution rate of API in the gastrointestinal fluids as a rate-limiting step. The improvement of dissolution rate of API will significantly increase absorption of poorly soluble drug in the systemic circulation.

Many strategies had been reported to enhance dissolution rates of IBS such as the formation of inclusion complexes, nanoparticles, and cocrystal [3], [4], [5]. One of the most popular techniques for modifying dissolution rates is the formation of a solid dispersion system of poorly soluble drugs with hydrophilic carriers [6]. According to BCS, the drug is considered very soluble when the absorption rate in humans is more than $90 \%$ of the given dose [7]. Irbesartan shows a low oral bioavailability of $26 \%$ so a method is needed to increase the solubility of the molecule [6].

Solid dispersion system is a dispersion of one or more active ingredients in an inert carrier or matrix in a solid-state which is prepared by solvent, melted, and combination of solvent and melted technique [8]. Solid dispersions are intended for solid product groups containing at least two different components, generally one hydrophilic carrier and one hydrophobic drug. Various carriers can be used in solid dispersion systems, including polyethylene glycol, polyvinyl pyrrolidone, urea, mannitol, and poloxamer [9], [10].

In this study, poloxamer 188 was used as a carrier. Poloxamer 188 is a nonionic copolymer consisting of ethylene oxide and propylene oxide which has been used to increase the solubility, dissolution, and bioavailability of drugs with poor solubility in water [11]. Poloxamer 188 was empirically chosen to prepare solid dispersions due to the low melting point (around $52-57^{\circ} \mathrm{C}$ ) and the nature of surfactants [12]. In 2009, Ghareeb et al. conducted a study on the preparation 
of a solid dispersion system for binary solutions from meloxicam using poloxamer 188 as a carrier [12].

In this study, the physicochemical properties of irbesartan in the form of solid dispersion will be studied using poloxamer 188 carrier by the solvent method. The solid dispersions were characterized using X-ray diffraction analysis, Fourier-transform infrared (FTIR) spectroscopy, and scanning electron microscopy (SEM). The dissolution rate profile of solid dispersions of irbesartan was determined using a Type II United States Pharmacopeia (USP) apparatus. The formation of solid dispersion and the results of analysis of solid dispersion systems are expected to improve the physicochemical properties of irbesartan.

\section{Materials and Methods}

\section{Materials}

Irbesartan (Dr. Reddy's) was kindly obtained as a gift from PT. Primatama Tata. Poloxamer 188, ethanol, and $\mathrm{HCl}$ were purchased from Merck (Germany). Paraffin liquid was purchased from PT. Harum Kimia (Indonesia).

\section{Methods} physical mixture

Preparation of solid dispersions system and

Solid dispersions of irbesartan-poloxamer 188 were prepared by the solvent co-evaporation technique. Irbesartan and poloxamer 188 were weighed according to the ratio of $1: 0.5 ; 1: 1$; and $1: 3 \mathrm{w} / \mathrm{w}$. Irbesartan and poloxamer 188 were dissolved with $96 \%$ ethanol until a clear solution was formed. The solution was evaporated and dried in a vacuum oven at $40^{\circ} \mathrm{C}$. The resulting solid is crushed, then passed through 60 mesh sieves and stored in a desiccator.

\section{Powder X-ray diffraction (PXRD) analysis}

The measurement conditions were as follows: Cu metal target, Ka filter, voltage 35-40 kV, and current $40 \mathrm{~mA}$, analysis carried out in the range of $2 \mathrm{q}$ at $5-35^{\circ}$. Intact irbesartan, poloxamer 188 , physical mixture, and irbesartan-poloxamer solid dispersion are placed on the sample holder (glass) and flattened to prevent particle orientation during sample storage.

\section{FT-IR spectrophotometry analysis}

Intact irbesartan, poloxamer 188, solid dispersion, and physical mixture were dispersed on attenuated total reflection which is pressed with high pressure. The absorption spectrum was recorded with FT-IR spectrophotometry at wave numbers $4000-400 \mathrm{~cm}^{-1}$.

\section{SEM analysis}

Intact irbesartan, poloxamer 188, irbesartanpoloxamer 188 solid dispersion, and physical mixture were placed on an aluminum sample holder. The surface morphology of each sample was then observed in various magnifications. Voltage was set at $20 \mathrm{kV}$ and $12 \mathrm{~mA}$ current.

\section{Dissolution rate profile}

The dissolution rate profile was performed by Type II USP apparatus. The equipment was adjusted at a speed of $50 \mathrm{rpm}$ and $900 \mathrm{~mL}$ dissolution medium of $0.1 \mathrm{~N}$ hydrochloric acid solution. The temperature was maintained at $37 \pm 0.5^{\circ} \mathrm{C}$. At pre-determined times (5; $10 ; 15 ; 30 ; 45$; and $60 \mathrm{~min}$ ), approximately $5 \mathrm{~mL}$ of aliquot were withdrawn and filtered. The concentration of irbesartan in the medium was determined by an ultraviolet-visible spectrophotometer at a maximum absorption wavelength of $245 \mathrm{~nm}$. The experiment was conducted in triplicate.

\section{Results and Discussion}

The results of X-ray diffraction analysis can be seen in Figure 1. The PXRD pattern showed that irbesartan has a high crystalline degree due to the presence of a number of distinctive sharp peaks at the diffraction angle $2 \theta=12.34^{\circ} ; 19.15^{\circ}$; and

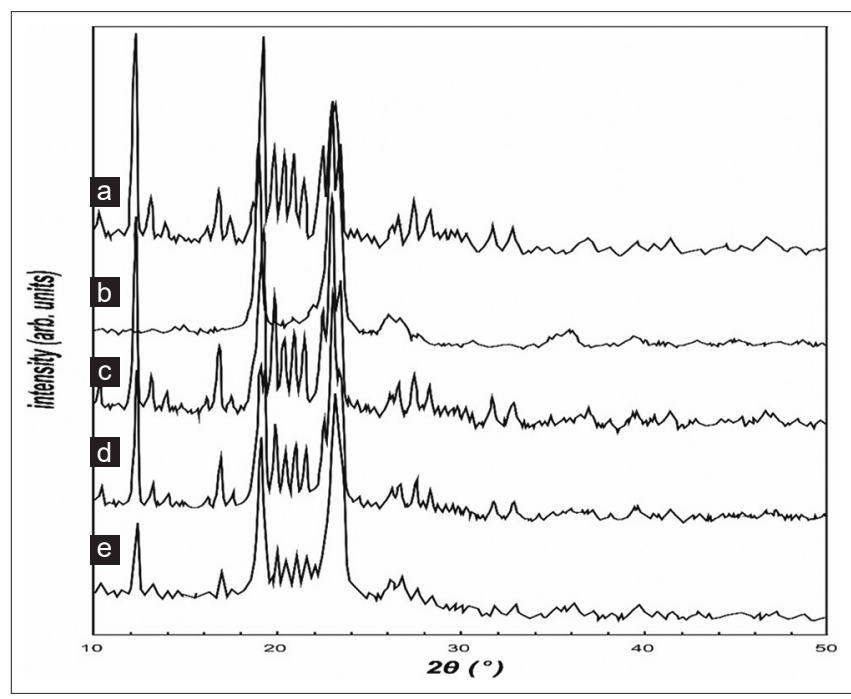

Figure 1: Powder X-ray diffraction pattern for (a) intact irbesartan, (b) poloxamer 188, (c) solid dispersion of irbesartan-poloxamer 188 at ratio 1:0.5, (d) solid dispersion of irbesartan-poloxamer 188 at ratio $1: 1$, (e) solid dispersion of irbesartan-poloxamer 188 at ratio 1:3 
$22.97^{\circ}$, while the poloxamer 188 diffraction pattern is semi-crystalline. The diffractogram pattern of solid dispersion of irbesartan-poloxamer 188 (1:0.5; 1:1; and 1:3) exhibited a decreasing in interference peak at diffraction angle $2 \theta=12.34^{\circ}$ (peak intensity is 6345,$9 ; 4705,5 ; 5439,3 ; 5197,1 ; 3077,1$; and 2915,3). The intensity of X-ray diffraction interference peak is calculated using WinPLOTR software.

In all solid dispersion samples of irbesartanpoloxamer 188 , there was also a decrease in intensity at several peaks but no new peaks were seen. Decreasing the intensity of the interference peak shows changes in the degree of crystallinity so that it will increase its solubility [13]. The results of PXRD analysis showed a decrease in the intensity of the interference peak at an angle of $2 \theta=12.34^{\circ}$ from 6345.9 to 2915.3 which indicates the formation of a crystal lattice that has a level of symmetry.

In the FT-IR spectra (Figure 2), intact irbesartan shows that there is a functional group $\mathrm{C}-\mathrm{H}$ at wave number $2953.87 \mathrm{~cm}^{-1}$, functional group $\mathrm{C}=\mathrm{O}$ at wave number $1729.32 \mathrm{~cm}^{-1}$, and functional group C-N at wave number $1613.71 \mathrm{~cm}^{-1}$. While the FT-IR spectra of poloxamer 188 show a wide peak at wave number $3503.67 \mathrm{~cm}^{-1}$ which shows the $\mathrm{O}-\mathrm{H}$ function group, the functional group $\mathrm{C}-\mathrm{H}$ at wave number 2877.71 and the functional group C-O at wave number $1095.14 \mathrm{~cm}^{-1}$. The results of this FT-IR analysis support the results of the previous analysis that the existence of peaks equal to the components of intact irbesartan and poloxamer 188 in all solid dispersion systems and can be proved to be the same as the physical mixed spectrum of irbesartanpoloxamer 188. This shows that there are no chemical interaction occurs between the two components of the solid dispersion constituent.

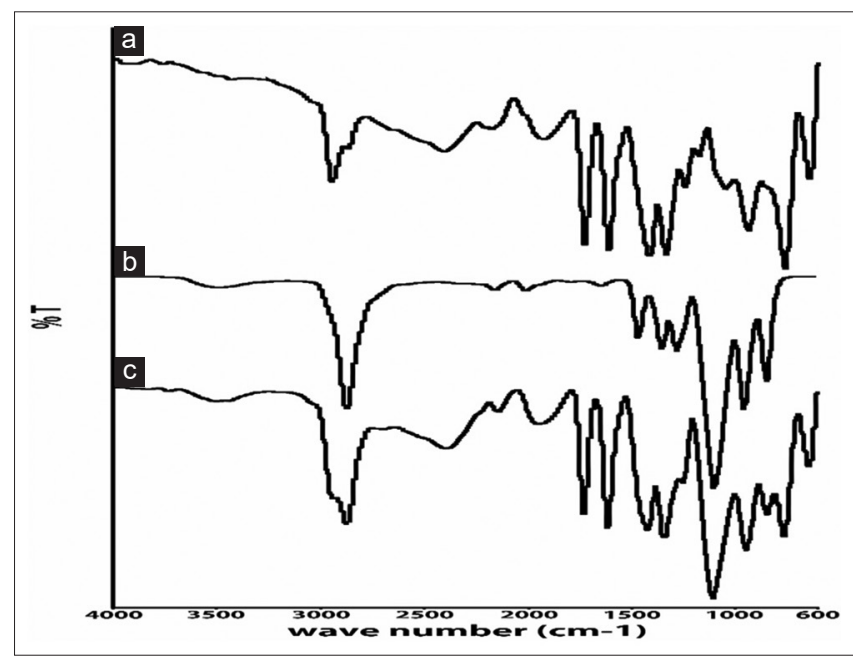

Figure 2: Fourier-transform infrared spectrum of (a) intact irbesartan, (b) poloxamer 188, (c) solid dispersion of irbesartan-poloxamer 188 at ratio 1:0.5

The results of morphological analysis by SEM (Figure 3) of irbesartan with a magnification of $2500 \times$ look like the surface of a particle that is roughly shaped while poloxamer 188 looks like a round particle with a smooth surface. The solid dispersion system of irbesartan-poloxamer 188 shows that a form of irregular particles dispersed molecularly forms an aggregate so that it cannot be distinguished from the shape of its constituent particles. This indicates that irbesartan has been dispersed in carrier polymers to form a solid dispersion system. The SEM result shows that the occurrence of physical interactions between these two substances that will affect the crystal morphology of each substance [13], [14].

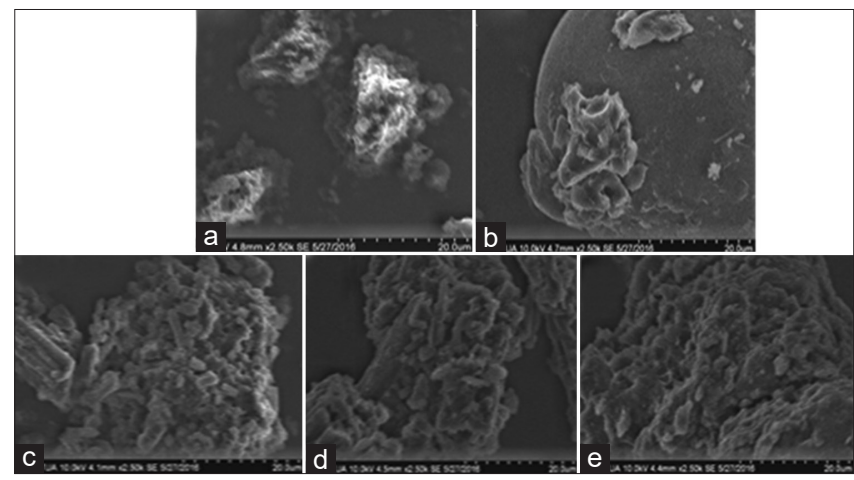

Figure 3: Scanning electron microscopy microphoto for (a) intact irbesartan, (b) poloxamer 188, (c) solid dispersion of irbesartanpoloxamer 188 at ratio 1:0.5, (d) solid dispersion of irbesartanpoloxamer 188 at ratio 1:1, (e) solid dispersion of irbesartan-poloxamer 188 at ratio $1: 3$

The dissolution rate profile (Figure 4) was carried out in $0.1 \mathrm{~N} \mathrm{HCl}$ dissolution medium with a speed of 50 RPM and tested for 60 min using the paddle method. This test is carried out by aliquot samples at $5,10,15,30,45$, and $60 \mathrm{~min}$. Irbesartan dissolution percentage results in the $60^{\text {th }}$ min for intact irbesartan, solid dispersion $1: 0.5,1: 1$, and $1: 3$, were $65.374 \%, 100 \%, 94.264 \%$, and $94.538 \%$. Based on the results obtained, the highest percentage of dissolved substances at minute 60 was shown to be a solid dispersion of 1 : 0.5 , which was completely $100 \%$. The increase in the amount of poloxamer 188 resulted in a decrease in the amount of irbesartan dissolved, this occurred due to poloxamer 188 which was surfactant so that irbesartan was trapped by poloxamer 188 so irbesartan was difficult to release. In addition, the increase in the amount of poloxamer 188 causes

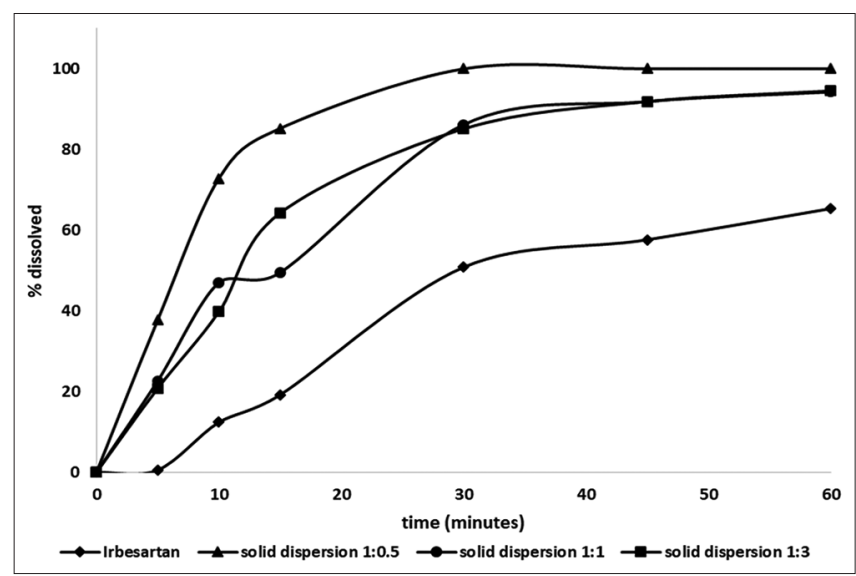

Figure 4: Dissolution profile for irbesartan and solid dispersion system 
the micelles to join to become aggregates of varying sizes [15].

According to the pharmacopeia, irbesartan must dissolve within 20 min not $<80 \%$ [16]. While in solid dispersion 1: 0.5 , irbesartan from solid dispersion dissolved approximately $85.240 \%$ in $15 \mathrm{~min}$, this indicates that the solid dispersion of irbesartanpoloxamer $188(1: 0.5)$ has met the requirements as stated in the literature.

\section{Conclusions}

From the current study, it can be concluded that the solid dispersion system of IBS with poloxamer 188 can modify the physicochemical properties of irbesartan such as decreasing the degree of crystallinity and surface morphology of substances. The solid dispersion system of irbesartan-poloxamer 188 can increase dissolution and highest dissolution obtained in a ratio of 1:0.5.

\section{Acknowledgment}

The author would like to thank PT. Tata Rasa Primatama for providing irbesartan.

\section{References}

1. Oparil S, Williams D, Chrysant SG, Marbury TC, Neutel J. Comparative efficacy of olmesartan, losartan, valsartan, and irbesartan in the control of essential hypertension. J Clin Hypertens (Greenwich). 2001;3(5):283-91, 318. https://doi. org/10.1111/j.1524-6175.2001.01136.x PMid:28718426

2. HayerAM. Finding solutions. Chem Eng News. 2010;88(22):13-8.

3. Hirlekar R, Kadam V. Preformulation study of the inclusion complex irbesartan-beta-cyclodextrin. AAPS PharmSciTech. 2009;10(1):276-81. https://doi.org/10.1208/s12249-009-9206-5

\section{PMid:19283492}

4. Zhang Z, Le Y, Wang J, Zhao H, Chen J. Irbesartan drug formulated as nanocomposite particles for the enhancement of the dissolution rate. Particuology. 2012;10(4):462-7. https://doi. org/10.1016/j.partic.2012.01.002

5. Haneef J, Chadha R. Antioxidant-based eutectics of irbesartan: Viable multicomponent forms for the management of hypertension. AAPS PharmSciTech. 2018;19(3):1191-204. https://doi.org/10.1208/s12249-017-0930-y

PMid:29247285

6. Kumar GA, Ram KC, Chaitanya C. Enhancement of solubility and dissolution rate of irbesartan by solid dispersion technique. Asian J Pharm Clin Res. 2011;4(2):36-40.

7. Chawla G, Bansal AK. A comparative assessment of solubility advantage from glassy and crystalline forms of a waterinsoluble drug. Eur J Pharm Sci. 2007;32(1):45-57. https://doi. org/10.1016/j.ejps.2007.05.111

\section{PMid:17618092}

8. Nijhawan M, Babu PR, Subrahmanyam CV. Cocrysta of irbesartan with hippuric acid. Indo Am J Pharm Res. 2015;5(4):1323-9.

9. Chiou WL, Riegelman S. Pharmaceutical applications of solid dispersion systems. J Pharm Sci. 1971;60(9):1281-302. https:// doi.org/10.1002/jps.2600600902

PMid:4935981

10. Serajuddin AT. Solid dispersion of poorly water-soluble drugs: Early promises, subsequent problems, and recent breakthroughs. J Pharm Sci. 1999;88(10):1058-66. https://doi. org/10.1021/js980403।

PMid: 10514356

11. Hang Y, Myung-Kwan C, Hoo-Kyun C. Preparation and characterization of piroxicam/poloxamer solid dispersion prepared by melting method and solvent method. J Korean Pharm Sci. 2007;37:1-5. https://doi.org/10.4333/kps.2007.37.1.001

12. Ghareeb MM, Abdulrasool AA, Hussein AA, Noordin MI. Kneading technique for preparation of binary solid dispersion of meloxicam with poloxamer 188. AAPS PharmSciTech. 2009;10(4):1206-15. https://doi.org/10.1208/s12249-009-9316-0

PMid:19862626

13. Zaini E, Wahyuni YS, Halim A, Yuliandra Y. Preparation of eutectic mixture of ketoprofen and nicotinamide for enhanced dissolution rate. Int J Pharm Sci Rev Res. 2015;35(1):161-4.

14. Alatas $F$, Soewandhi SN, Sasongko L, Ismunadar UH, Uekusa $\mathrm{H}$. Cocrystal formation between didanosine and two aromatic acids. Int J Pharm Pharm Sci. 2013;5(3):275-80.

15. Wagh VT, Jagtap VA, Shaikh TJ, Nandedkar SY. Formulation and evaluation of glimepiride solid dispersion tablets for their solubility enhancement. J Adv Sci Res. 2012;3(4):36-41.

16. Departemen Kesehatan Republik Indonesia, Farmakope Indonesia Edisi V, Direktorat Jendral Pengawasan Obat dan Makanan: Jakarta; 2014. 